\title{
LA DERIVACIÓN DE SUSTANTIVOS A PARTIR DE PARTICIPIOS
}

Todas las gramáticas españolas mencionan el tema de la derivación, pero ninguna lo trata exhaustivamente y con miras a dar una explicación sistemática del fenómeno; algunas incluso renuncian explícitamente a abordarlo ${ }^{1}$. Filólogos y lexicólogos estudian afijos aislados o microsistemas de afijos sin intentar, por lo general, una formulación que abarque la totalidad de éstos y su funcionamiento en la lengua ${ }^{2}$.

Si se confrontan diversas listas de afijos españoles -en particular de sufijos-, llama la atención la falta de coincidencia, no sólo en cuanto al número de elementos que abarcan, sino también en cuanto a la forma con que aparece un mismo sufijo en las diferentes enumeraciones: lo que para unos es -able, -ible, para otros se reduce a -ble; lo que para unos es -adero, -edero, -idero, para otros es -dero y hasta -ero ${ }^{3}$. Incluso aparecen en un mismo inventario los mismos sufijos bajo diferentes formas, que entonces se relacionan por medio de referencias cruzadas (por ejemplo, GRAE, p. 131: -able, véase -ble). Todo esto obliga a preguntarse por los criterios seguidos en la separación de base y sufijo.

En un intento de inventariar los sufijos contenidos en un corpus ${ }^{*}$ con ayuda del análisis de las palabras pretendidamente sufija-

1 Véase, por ejemplo, Salvador Fernández Ramírez, Gramática española, Madrid, 1951, p. xiii, y ahora J. Alcina Franch y J. M. Blecua, Gramática española, Barcelona, 1974.

2 Uno de los trabajos más completos sigue siendo la obra de JosÉ AlemanyBolufer, Tratado de la formación de palabras en lengua castellana, BRAE, ts. 4,5 y 6 (1917-1919).

3 Cuestionario para el estudio coordinado de la norma lingiiistica culta de las principales ciudades de Iberoamérica y de la Peninsula ibérica, t. 2, Morfosintaxis, Vol. 1, PILEI, Madrid, 1972; Manuel Seco, Gramática esencial del español, Madrid, 1973, pp. 189-196; Real Academia Española, Gramática de la lengua española, 1931, pp. 131-146 (abreviaré $=$ GRAE); id., Esbozo de una nueva gramática de la lengua española, Madrid, 1973, pp. 165-166 (abreviaré: Esbozo); Rufino José Cuervo, “Apuntaciones críticas", Obras, t. 1, pp. 772-810.

4 Tomé las 1000 primeras palabras de cada una de las muestras contenidas en El habla de la ciudad de México: materiales para su estudio, México, 1971. 
das en constituyentes inmediatos, es decir, en base y derivativo, pude darme cuenta del problema de fondo al que responde esta multiplicidad de presentación.

La derivación se define como el procedimiento a través del cual se forman palabras nuevas a partir de palabras ya existentes ${ }^{5}$. No se suele aclarar, sin embargo, cuál es el concepto de palabra presupuesto en esta definición. ¿Se trata de palabra en el sentido de representante de todo un paradigma o de un determinado elemento de este paradigma?

Según GRAE ( $\$ 177 a)$, "Consiste la derivación en formar palabras nuevas por medio de sufijos que se añaden al radical de un vocablo que tiene existencia independiente en la lengua..." (el subrayado es mío) ${ }^{6}$.

Ahora bien, al hablar del verbo se mencionan en $G R A E$ varios radicales: "En los tiempos simples debemos distinguir dos radicales: una constituida por las letras que preceden a la terminación -ar, -er, o -ir del infinitivo... y otra formada por el mismo infinitivo" ( $\$ 86 b)$. Del inciso $d$ del mismo párrafo se desprende, además, la existencia de un tercer radical para los tiempos compuestos:- el participio en su forma invariable. Esto parece indicar que en opinión de GRAE el derivado se forma a partir de un elemento determinado del paradigma con su radical correspondiente ${ }^{7}$. Bajo este enfoque es indispensable determinar en cada caso de cuál de todas las formas se trata y qué modificaciones sufre en el proceso de derivación.

Cabe recordar aquí que las más diversas formas de la conjugación verbal han dado lugar a la creación de sustantivos por el procedimiento de habilitación. Menéndez Pidal en su Manual de gramática histórica española (Madrid, 1966), menciona como fuentes verbales de nuevos sustantivos en orden de importancia, a) participios pasados; $b$ ) infinitivos, $c$ ) las otras formas verbales. Me parece que se pueden identificar también formas específicas de la conjugación verbal como bases de derivados (casos obvios serían, por ejemplo, callandito, hechizo), y que es necesario hacerlo para llegar

5 Bello dice “[Palabras] derivadas son las que nacen de otras de nuestra lengua, variando de terminación como regularmente sucede, o conservando la misma terminación, pero añadiendo siempre alguna nueva idea" (Gramática de la lengua castellana, 7a ed., Buenos Aires, 1964, p. 53, § 87) .

6 En $\$ 5$ vocablo está definido como sinónimo de palabra.

7 En el Esbozo no hay reglas específicas de derivación, pero se dice que "Si suprimimos de una palabra nominal todos los sufijos, lo que queda se denomina radical o raíz, especialmente cuando es parte de una forma verbal, como en: ven-id-ero, quer-e-ncia, salv-a-mento, holg-orio y más frecuentemente tema o base de derivación en los otros casos..." $(\S 2.1 .2 b)$. Es decir, se parte de una forma verbal específica, aunque no se explica el por qué de esta segmentación ni cómo se forman nuevos derivados. 
a una interpretación inequívoca del proceso de derivación, así como para explicar el valor semántico y el comportamiento sintáctico del derivado.

Creo haber encontrado en mi análisis de los textos del Habla... un grupo de sufijos relacionados entre sí por su significado y por la forma verbal a que se añaden y son los que estudié con cierto detalle. Intenté - a través del reconocimiento de la base-delimitar los sufijos, describir los cambios fonológicos que se producen en el proceso y traté, finalmente, de describir cada sufijo en su forma, comportamiento y valor semántico aproximado.

Al analizar los textos buscaba aquellos casos donde se hubiera producido un cambio formal, esto es, la añadidura de un elemento ligado pospuesto que no perteneciera a ningún paradigma de morfemas gramaticales. Menciono, sin embargo (cf. infra, p. 327), casos en los que la derivación se puede interpretar como ocurrida a través de la pérdida de un elemento y, en otros, a través de la existencia del sufijo $\phi$. No tomé en cuenta la habilitación, o sea el simple cambio de función o significado no asociado a cambio formal, pues me interesaba sobre todo identificar y delimitar los sufijos. Por esta razón no recopilé los sustantivos terminados en -ado, -a; -ido, -a. Descarté también aquellas palabras que parecen tener sufijos pero cuyo primer elemento no tiene hoy existencia autónoma en la lengua. Esto equivale, más o menos, a descartar las formaciones antiguas.

Para la identificación de los sufijos he seguido el procedimiento de comparar palabras formalmente semejantes y con fondo semántico común con la palabra que, se supone, es la derivada. En el caso de los postverbales son muchas las formas de la conjugación que cumplen esa condición. Si tenemos por ejemplo el derivado educación, podemos oponerlo a:

$\begin{array}{ll}\text { educa-r } & \text { educ-ó } \\ \text { educa-ndo } & \text { educa-ba } \\ \text { educa-do } & \text { educa-ste } \\ \text { educa-s } & \text { educa-ré [etc.] }\end{array}$

Si al revisar las ocurrencias del sufijo -ción nos encontramos con casos como presunción, convicción, distinción, para los que se ofrece como base un elemento calificado por los diccionarios como participio irregular, esto nos puede llevar a preferir, también en los otros casos, el participio como base. Pero queda, además, el recurso de comparar los contextos donde aparece el sustantivo derivado con los contextos en que aparecen las formas verbales. Se trataría entonces de seleccionar como base aquella forma de la conjugación que se relaciona con el mayor número de construcciones en que se 
encuentra el derivado. Por ejemplo, en construcciones del tipo: "La distribución de desayunos escolares por las autoridades fue eficiente", el participio es la única forma de la conjugación que puede entrar en un contexto semejante, y precisamente en su función más característica: la de formar la voz pasiva ${ }^{8}$. Es decir: "Los desayunos escolares fueron distribuidos eficientemente por las autoridades". El sujeto agente aparece en ambas oraciones, aun cuando en la primera su función sintáctica es la de un complemento adjetivo. $\mathrm{A}$ mi modo de ver, esto indica que el participio aún está presente en el derivado.

No pretendo decir con esto que todos los derivados de participios procedan del participio en construcción pasiva, sino, simplemente, que el participio es el más apto para cubrir todos los casos, incluyendo las construcciones sustantivo + complemento equivalente de sujeto agente.

Hay también numerosos derivados de verbos intransitivos: por ejemplo, colaboración, conversación, que se relacionarían con construcciones en las que el participio aparece en un tiempo compuesto. Parece confirmar esto el hecho de que en estos sustantivos se conserva la idea de algo concluido: el valor perfectivo propio de los tiempos compuestos. En estos sustantivos el sufijo que en unos casos significa 'acción y efecto de' pasa a significar 'efecto de' y más aún, esos sustantivos se convierten en nombres abstractos. (Más adelante volveré sobre la discusión de los valores semánticos de los sufijos aquí tratados según la base a que se añaden).

Tomar el participio como base de derivación de ciertos sustantivos postverbales significa máxima economía en su descripción, ya que a) no se necesitan aclaraciones ni reglas especiales para explicar la $i$ en los derivados que proceden de verbos en -er; $b$ ) es más económico derivar, por ejemplo, impresión de impreso y otros derivados de participios irregulares del participio correspondiente, que de cualquier otra forma verbal; c) puede proponerse como un solo sufijo por ejemplo -ción en vez de reconocer -ación, -ición que aparecen en algunas listas.

Por todo lo anterior, decidí analizar las ocurrencias ${ }^{9}$ de los sustantivos en -ción (-sión, -ión), -miento (-mento), -ura y de todos

8 Se puede decir que en construcciones de este tipo se sobreentiende un verbo, o más bien el participio de hacer en su forma variable. No me parece válida esta objeción, pues, aunque encontramos oraciones de esta forma con participio explícito, en realidad se trata de una redundancia, ya que para el hablante el valor de -ción y otros sufijos (que trataré de analizar más adelante) es precisamente el que señalan los diccionarios en las definiciones: 'acción y efecto de'.

9 Para confirmar alguna regularidad observada en los ejemplos (o cuando resultaba insuficiente mi material), he usado $A$ reverse dictionary of the Spanish language, de Fred A. Stahl y Gary E. A. SCAvNicky, Urbana, lll., 1973. 
aquellos sustantivos postverbales en los que encontraba un componente semántico igual al expresado por estos sufijos, bajo la hipótesis de que se trata de derivados de participios. Estos son los resultados:

-CIÓN

De 136 sustantivos diferentes (306 ocurrencias) que registré con este sufijo, 83 se derivan de participios regulares mediante pérdida de -do, -da y añadidura de -ción; en todos los casos el acento pasa de la palabra base al sufijo. Queda como marca del participio que sirvió de base la vocal de la penúltima sílaba. 79 sustantivos proceden de participios de verbos en -ar y 4 de verbos en -ir:

$\begin{aligned} \text { alimenta-do } & \text { alimentación } \\ \text { capta-do } & \text { captación } \\ \text { civiliza-do } & \text { civilización } \\ \text { compara-do } & \text { comparación } \\ \text { educa-do } & \text { educación } \\ \text { forma-do } & \text { formación } \\ \text { defini-do } & \text { definición } \\ \text { exhibi-do } & \text { exhibición } \\ \text { nutri-do } & \text { nutrición }\end{aligned}$

Registré tres casos en los cuales la relación semántica entre la posible base y el derivado es discutible: vegetación que parece estar hoy semánticamente alejado de vegetar aunque el Manual de la Real Academia Española (1950) dice: "acción y efecto de vegetar"; generación que el Manual define como "acción y efecto de engendrar" (de la entrada generar remite a engendrar), y oración, pues el verbo orar hoy sólo se usa con el valor de 'rezar'.

Hay, además, cinco sustantivos que tienen varias posibilidades de análisis en constituyentes inmediatos, sin que en una visión puramente sincrónica y distribucional, se pueda dar preferencia a alguna de ellas. Se trata de desintegración, desorientación, insubordinación, predisposición, sobreprotección que se pueden describir como formados por:
a) des + integración
in + subordinación
pre + disposición
sobre + protección

es decir, como prefijados

b) desintegra- (do) + -ción desorienta- (do) + -ción insubordina- (do) + -ción etc. 
o sea, como sufijados, o

c) des + integra-(do) + -ción

in + subordina- (do) + -ción

pre + dispues- (to) + -ción [etc.]

es decir, como parasintéticos.

En las interpretaciones $a$ ) y $b$ ) se estarían considerando los sustantivos como derivados secundarios o de segundo grado; mientras que en la interpretación $c$ ) serían derivados de primer grado. La búsqueda de relaciones semánticas nos puede inclinar a favor de una u otra interpretación, pero sólo el estudio diacrónico daría cuenta del proceso real.

Para 12 sustantivos más se observan cambios fonológicos ocurridos en el proceso de derivación:

\section{-i- epentética}

El derivado de poner y los derivados de verbos que a su vez derivan de poner presentan una -i- epentética cuya presencia se podría explicar diciendo que sirve para conservar la identidad de la base. El mismo fenómeno se observa para los sustantivos terminados en -quisición (adquisición, requisición, disquisición). Ahora bien, si se toma en cuenta la perspectiva histórica, se ofrece * pósito como base, elemento que se encuentra en depósito y expósito.

Enumero los casos extraídos del corpus que proceden de poner y sus derivados:

$$
\begin{aligned}
\text { compues-to } & \text { composición } \\
\text { dispues-to } & \text { disposición } \\
\text { expues-to } & \text { exposición } \\
\text { pues-to } & \text { posición } \\
\text { predispues-to } & \text { predisposición } \\
\text { transpues-to } & \text { transposición }
\end{aligned}
$$

obviamente se observa también que ue se convierte en $o$ al perder el acento tónico.

\section{-u- epentética}

Sólo encontré un caso para este fenómeno (aunque se podría asociar a solución) que eliminé porque no cumplía con el requisito de la autonomía del primer elemento: revuel-to revolución (donde también ue $>$ o). Es probable, sin embargo, que el sustantivo del cual el $D R A E$ y Corominas derivan revolución (revolutio, -onis), sea, a su vez, derivado de participio latino. Quizá, disoluto sea tes- 
tigo de esta evolución en la lengua actual. También presentan -ulos demás sustantivos procedentes de volver y *solver en derivación secundaria.

$u i>u$

Encontré que, con regularidad, los participios de verbos en -uir pierden la -i- del diptongo de la raíz en el proceso de derivación:

$$
\begin{array}{cl}
\text { constitu (-i-do) } & \text { constitución } \\
\text { disminu (-i-do) } & \text { disminución } \\
\text { institu (-i-do) } & \text { institución }
\end{array}
$$

También aquí se ofrece una interpretación histórica: institu-to institución.

\section{Pérdida de -i- de la desinencia del participio}

Se pierde esta vocal en los derivados de tener y venir de los que sólo documenté dos ejemplos: entreten-i-do, entretención, interven -i-do, intervención.

Pérdida de -ec- ante -ción

Este cambio se observa en los sustantivos que provienen de verbos en -ecer. Sólo documenté: apar-ec-i-do aparición ${ }^{10}$. Del resto de los sustantivos terminados en -ción que registré, 18 no cumplen con el requisito de la autonomía de la base, es decir no existe en la lengua actual el verbo que pudo haber dado origen al sustantivo en cuestión. Los verbos de la lengua actual relacionados con doce de ellos son derivados de sustantivos, por ejemplo, accionar, ambicionar, condicionar, emocionar, etc.

Encontré cuatro ejemplos para los que los diccionarios declaran participios irregulares (sin aclarar de qué verbos), que podrían tomarse como bases para derivar estos sustantivos. Es decir aparentemente cabría derivarlos de estos participios, sin que se pierda el carácter verbal de la base: absorción, atención, excepción, sujeción. Me parece, sin embargo, que los participios irregulares correspondientes no se usan con carácter verbal y que los verbos actuales relacionados con dos de estos sustantivos provienen, a su vez, de participios latinos (exceptuar, sujetar).

10 De los verbos en -ecer se suelen formar sustantivos en -miento, por ejemplo, agradecimiento, fallecimiento, acontecimiento. Hay, sin embargo, algunos casos en -ción y a éstos me refiero aquí. Otro ejemplo tomado del diccionario inverso: fornecer fornición. 
En el corpus recogi un grupo de veinte sustantivos para los que resulta antieconómico intentar una derivación a partir de los participios o de cualquier otra forma de los verbos cuyos significados se relacionarian con esos sustantivos. En este caso pienso en dos soluciones:

a) Desechar el sustantivo, es decir, declarar que no es derivado porque no cumple con el requisito del primer elemento autónomo. Hay, en la lengua actual, en muchos casos adjetivos o sustantivos que por su similitud formal y semántica invitan a ser tomados como base de esos derivados. Pero me parece que si tomamos esas bases, no reconoceriamos la tendencia predominante de la lengua que es la de añadir este sufijo a la base verbal. Por lo menos en la actualidad, no se dan casos de derivación a partir de adjetivos no participiales.

b) Suponer aquí supletivismo de la raíz. Pueden ser cultismos (audición) o, en la perspectiva histórica, estos sustantivos se revelan como derivados de participios latinos. Enumero algunos ejemplos y los términos más semejantes del léxico actual:

$\begin{aligned} \text { atracción } & \text { atractivo } \\ \text { audición } & \text { audible } \\ \text { construcción } & \text { constructivo, constructor } \\ \text { convicción } & \text { convicto } \\ \text { dirección } & \text { directo, director } \\ \text { infección } & \text { infecto } \\ \text { instrucción } & \text { instructivo, instructor } \\ \text { producción } & \text { producto, productivo, productor } \\ \text { proyección } & \text { proyector, proyectar, etc... }\end{aligned}$

En resumen, se puede decir que -ción es un sufijo tónico heterogéneo ${ }^{11}$, que se utiliza para formar sustantivos femeninos a partir de participios de las tres conjugaciones y admite después de él -al, -ar (y todas las desinencias de la primera conjugación), -ada, -ario, -ero, -ismo, -ista, -aza y el morfema de plural. Su significado aproximado es 'acción y efecto de'.

No documenté ejemplos de sustantivos derivados mediante el sufijo -zón, pero probablemente se puedan encontrar sufijados con este elemento que sean postparticipiales y donde el sufijo tenga el mismo valor que -ción (matazón, quemazón).

-IÓN

Se pueden distinguir dos tipos de derivados en -ión: a) los que provienen de participios irregulares en -so (-sión) ; b) los que pro-

11 Llamo homogéneos a los afijos que producen palabras de la misma clase que la base y heterogéneos a los que provocan cambio de clase. 
vienen de participio regular. Del primer caso (a) registré 19 sustantivos diferentes en -sión ( 55 ocurrencias) de los cuales cinco no tienen verbo base correspondiente en la lengua actual (cohesión, excursión, ilusión, ocasión, presión) y tres son, presumiblemente, prefijados (revisión, subdivisión, televisión). Para los restantes podemos encontrar un participio irregular, aunque casi siempre sin uso verbal. Enumero algunos derivados y los participios correspondientes:

$\begin{aligned} \text { conclus-o } & \text { conclusión } \\ \text { confes-o } & \text { confesión } \\ \text { divers-o } & \text { diversión } \\ \text { expres-o } & \text { expresión } \\ \text { impres-o } & \text { impresión } \\ \text { precis-o } & \text { precisión } \\ \text { tens-o } & \text { tensión }\end{aligned}$

En el segundo caso $(b)$, de los cinco sustantivos diferentes registrados (12 ocurrencias), tres quedaron descartados porque no existe un verbo actual correspondiente. Los dos restantes se pueden derivar del participio suponiendo pérdida de la desinencia de éste. Se trata de unión y opinión. (Véase infra, sustantivos postverbales en $-0,-a,-e)$.

\section{-MIENTO}

De 22 sustantivos diferentes (61 ocurrencias) no deseché ninguno. Se reparten como sigue entre las tres conjugaciones: 8 provienen de participios de verbos en -ar, 9 de participios de verbos en -er y 5 de participios de verbos en -ir. En todos los casos se pierde la terminación -do y siempre se presenta cambio del acento de la base al sufijo; es decir, el sufijo es tónico. El resultado de la sufijación con -miento es siempre un sustantivo masculino. Por ejemplo:

$$
\begin{aligned}
\text { trata-do } & \text { tratamiento } \\
\text { mejora-do } & \text { mejoramiento } \\
\text { manteni-do } & \text { mantenimiento } \\
\text { procedi-do } & \text { procedimiento } \\
\text { surgi-do } & \text { surgimiento } \\
\text { senti-do } & \text { sentimiento } \\
\text { cumpli-do } & \text { cumplimiento }
\end{aligned}
$$

En dos casos (descubrimiento y desenvolvimiento) tuve que suponer la restitución del participio regular. Estos dos sustantivos y renacimiento pueden analizarse como prefijados, sufijados o parasintéticos en primera o segunda derivación: renacimiento < renacido, nacimiento, nacido; preferi tomar renacido como base. 
En el mismo sentido decidí los casos de descubrimiento y desenvolvimiento donde me parece aún más clara la procedencia de *descubrido y *desenvolvido respectivamente. Marco estas formas como hipotéticas, aunque se pueden documentar en lenguaje infantil o rural. El derivativo -miento se podría caracterizar del siguiente modo: es un sufijo tónico, heterogéneo que se utiliza para formar sustantivos masculinos a partir de los participios de las tres conjugaciones y que no admite después de él más morfema que el de plural. Su significado es 'acción y efecto de'.

-MENTO

Registré 17 ocurrencias (sólo siete sustantivos diferentes; deseché cuatro porque no cumplían con el requisito de la autonomía del primer elemento) de derivados en -mento, sufijo que podría considerarse variante de -miento (en el $D R A E$, por ejemplo, se declaran sinónimos pedimiento y pedimento). Conservé campamento, que tiene la peculiaridad de relacionarse con un verbo creado como parasintético y cuyo participio en este nuevo proceso de derivación pierde también el prefijo: a-campa-do campamento; conservé también fundamento y predicamento por tener los verbos correspondientes: fundar y predicar.

Al contrario de lo que se ha dicho para -miento el sufijo -mento admite diversos sufijos después de él, por ejemplo -al, -ar, -ario (fundamental, fundamentar, reglamentario).

-URA

Se observa duplicidad de funciones en el caso de este sufijo. Unas veces -ura se utiliza para formar sustantivos a partir de adjetivos y otras a partir de participios. Toma entonces este sufijo dos valores distintos: en el primer caso 'cualidad de' y en el segundo 'acción y efecto' o, frecuentemente, 'efecto de'. Se obtienen así dos series:

$$
\begin{aligned}
& \text { Derivados de adjetivos } \\
& \text { cuerd-o cordura } \\
& \text { llan-o } \text { llanura } \\
& \text { hermos-o } \text { hermosura } \\
& \text { alt-o } \text { altura }
\end{aligned}
$$

$$
\begin{aligned}
\text { Derivados de } & \text { participios } \\
\text { hech-o } & \text { hechura } \\
\text { vestid-o } & \text { vestidura } \\
\text { escrit-o } & \text { escritura } \\
\text { cubiert-o } & \text { cobertura }
\end{aligned}
$$

Incluso se da algún caso de derivación homogénea con este sufijo: diablo: diablura. En ocasiones el valor del sufijo en los derivados de participio coincide con el de los derivados de adjetivos, por ejemplo, suel-to soltura. Esto hace suponer que fueron derivados 
del participio en su función adjetiva. En otros casos, sin embargo, se conserva claramente el carácter verbal. Pocas veces se construye con complemento adjetivo equivalente de sujeto agente; tal vez se construya con mayor frecuencia con el equivalente del complemento directo bajo la forma de complemento adjetivo introducido por 'de':

Conmovió al auditorio la lectura de los poemas por el autor.

Causó consternación la ruptura de relaciones entre los dos países.

Podría decirse también: "Causó consternación el rompimiento de relaciones entre los dos países" (formado sobre el participio regular).

En el caso de ruptura parece haber una especialización frente a rotura que se emplea para designar 'el efecto de' mientras que sólo ruptura se emplea en el sentido de 'acción'; o sea que en el ejemplo anterior no podemos decir *rotura de relaciones. La explicación podría ser que el adjetivo es roto.

De las 93 ocurrencias de -ura en sustantivos derivados que registré, sólo 18 (5 sustantivos diferentes) son postparticipiales: dos de verbos en -ar, dos de verbos en -ir, uno de verbo en -er. En todos los casos se pierde la vocal final del participio en el proceso de derivación y siempre el acento pasa de la base al sufijo. El resultado es, en todos los casos, un sustantivo femenino:

$$
\begin{aligned}
\text { escrit-o } & \text { escritura } \\
\text { suelt-o } & \text { soltura } \\
\text { vestid-o } & \text { vestidura }
\end{aligned}
$$

Los cambios fonológicos que se observan son: $u e>o$ en los participios irregulares al perder la base el acento; $i>e$ para derivados de verbos en -er. El carácter verbal se percibe más en los casos de pintura y escritura aunque no documenté ejemplos de uso con complemento adjetivo equivalente a sujeto agente ni a complemento directo.

El derivativo -ura en postverbales se podría caracterizar de esta manera: es un sufijo tónico, heterogéneo, que se emplea para formar sustantivos femeninos a partir de participios de verbos de las tres conjugaciones y que admite después de él: -ar, -al, -ario, -ero, -era, -ía (escriturar, postural, escriturario [DRAE], costurero, costurera, pagaduria) y el morfema de plural. Su significado puede ser 'acción y efecto de', o, con mayor frecuencia, 'efecto o resultado de'. De allí el sustantivo pasa frecuentemente a ser nombre de un objeto o expresión de un concepto. 
-ADO, -IDO

En la primera parte de este trabajo aclaré que en mi recuento de palabras sufijadas no quedaron incluidas las en -ado, -a; -ido, -a. Gon esto creí eliminar participios en función adjetiva y habilitados como sustantivos. Sin embargo, al penetrar más en el problema de los sustantivos postverbales, y al hacer un análisis crítico de algunos ejemplos, me di cuenta de la existencia de sustantivos de esta forma que son resultado de un verdadero proceso de nominalización verbal y que tienen en común con los analizados anteriormente el significado 'acción y efecto de' aunque con frecuente especialización en uno de estos valores. Si comparamos por ejemplo:

El hombre herido fue operado de emergencia.

El herido fue operado de emergencia.

Las tropas han entrado al pueblo.

La entrada de las tropas al pueblo.

percibimos claramente que se trata de dos procesos distintos. En un caso está en la base el participio en uso adjetivo; en el otro, en uso verbal.

Cabe hablar de habilitación de una de las formas del participio variable, aunque preferí distinguir los dos procesos, considerando que en el primer caso se trata de sustantivación o habilitación de un adjetivo y en el segundo de derivación; sobre todo, porque para hablar de habilitación en el segundo caso se estaría partiendo de formas hipotéticas para algunos sustantivos con forma de participios femeninos (por ejemplo, llegada).

Supongo la existencia de un sufijo $\phi$ para señalar que los sustantivos derivados coinciden con los demás tratados en este trabajo en aquella parte de su contenido expresado por -miento, -ción, -ura, etc., sin que tengan morfema o significante correspondiente. Supongo, además, que este sufijo equivaldría a un sustantivo masculino o femenino con el que concuerda el participio en cuestión: sufijo $\phi$ femenino + llegado = llegada.

Los ejemplos registrados de este proceso, que documenté insuficientemente por las razones antes expuestas son:

A su llegada va viendo...

¿Qué invento de jugadas haces!

La evaporación, el secado, la transferencia...

Es decir todos proceden de participios de verbos en -ar. Otros ejemplos podrían ser: leida, lavada, helada, caida, cuidado, revelado.

Se puede decir entonces que los derivativos $\phi$ femenino y masculino añadidos a participios son heterogéneos, y se utilizan para for- 
mar sustantivos a partir de participios de las tres conjugaciones. No admiten más morfema que el de plural. Su significado aproximado es 'acción y efecto de'.

$-\mathrm{O},-\mathrm{A},-\mathbf{E}$

Para otro gran grupo de sustantivos postverbales también se ofrecen dos posibilidades de derivación. Se trata de los que frecuentemente se han llamado postverbales en -o, -a, -e. Me interesan aquí los que provienen de los llamados verbos de acción, como por ejemplo rescatar:

Los heridos fueron rescatados por la Cruz Roja.

El rescate de los heridos por la Cruz Roja.

Cuervo (op. cit., p. 804) llama a estos sustantivos "derivados retrógrados" y señala que se trata de un procedimiento heredado del latín y sumamente fecundo en las lenguas romances.

A causa de la completa identidad formal de sustantivos primitivos y derivados, resulta que, en sincronía, es imposible hablar de derivación en uno u otro sentido. En esta perspectiva no hay razón para suponer: pago $>$ pagar, pagado $>$ pago. Me limito por ello a señalar la existencia del procedimiento y la posible conveniencia de derivar también aquí del participio para explicar el comportamiento sintáctico de estos sustantivos. Se podría decir entonces que hay derivación mediante pérdida de la desinencia del participio, aclarando que los derivados toman alguno de los morfemas del sustantivo: -o, -a, -e. Es decir, se pueden considerar estos sustantivos como resultado de una verdadera derivación retrógrada ${ }^{12}$, o $(\phi)$ masculino o femenino suponiendo entonces que en el proceso de derivación el participio pierde su desinencia -ado, -a, -ido, -a:

El preso se ha fugado.

La fuga del preso.

Este último planteamiento daría mayor homogeneidad al grupo de los sustantivos postverbales con valor 'acción y efecto de' al considerar también sufijados a los de este grupo.

Solamente quiero mencionar aquí otros dos tipos de formaciones nominales que a primera vista podrían parecer postparticipiales pero que no lo son pues carecen de base verbal. Unos están forma-

12 Es de tenerse en cuenta que este procedimiento compite con uno que da por resultado postverbales terminados en consonante, es decir postverbales que después de perder la desinencia no tomaron morfema de género como, por ejemplo, desliz, desdén. 
dos sobre el modelo del participio como, por ejemplo, colorido, sustantivo y colorado, adjetivo; otros -derivados homogéneos- deben su parecido formal con los participios al sufijo polisémico -ada (fachada, carretada, muchachada, lanzada).

Algunos autores (Cuervo, Corominas) mencionan otro procedimiento de derivación postparticipial mediante pérdida de -ad- (todos los ejemplos que dan proceden de verbos en -ar), como por ejemplo:

$$
\begin{aligned}
\text { caducado } & \text { caduco } \\
\text { anexado } & \text { anexo } \\
\text { salvado } & \text { salvo }
\end{aligned}
$$

que no tomo en cuenta aquí porque, al parecer, son adjetivos los que se forman así, aunque, desde luego, con la posibilidad de sustantivación ulterior, por ejemplo:

$$
\begin{aligned}
& \text { un escrito anexado } \\
& \text { un escrito anexo } \\
& \text { un anexo }
\end{aligned}
$$

Finalmente, quiero hablar de un grupo de sufijos que ocasionalmente pueden producir postverbales con valor 'acción y efecto de' y para los que documenté muy pocos o ningún ejemplo:

\section{- (A) TO}

Este sufijo, que, según la Academia, es la forma erudita de -ado, existe como formador de nombres de cargos o profesiones (literato, decanato), como derivativo homogéneo en los términos científicos, sobre todo de la química (sulfato, clorato, etc.), y en algunos postverbales con valor 'acción y efecto', por ejemplo, asesinato, mandato. Parece que en ellos predomina la idea de algo concluido, de 'efecto de', aunque ocasionalmente los podemos encontrar acompañados de complemento adjetivo equivalente al sujeto agente del verbo correspondiente: "El asesinato del padre por el hijo horrorizó a todos". (No documenté ejemplos).

-(A) JE

La presencia del sufijo -aje en derivados homogéneos del tipo vasallaje, bandidaje, voltaje, engranaje, personaje, hace que se separe de igual modo el de los postverbales procedentes de verbos en -ar. Parece, sin embargo, que la -a- corresponde a la letra temática de la primera conjugación, aunque no encontré ejemplos derivados de verbos de las otras conjugaciones ni en - (a) je, ni en *-ije. Como 
posible postverbal sólo documenté doblaje; todos los demás sustantivos en -aje que registré son derivados de sustantivos. Podemos, sin embargo, ejemplificar el proceso de derivación postverbal con los siguientes: rodaje, embalaje, montaje, aterrizaje, almacenaje, y derivarlos del participio correspondiente diciendo que en el proceso se pierde $-d o$ :

$$
\begin{array}{cl}
\text { embala-do } & \text { embalaje } \\
\text { monta-do } & \text { montaje }
\end{array}
$$

-ERO, -A

Se trata del más polisémico de los sufijos y de uno de los más productivos del español. Con base verbal, participial, ha producido adjetivos y sustantivos: a) nombres de lugar (matadero, bebedero); b) de instrumento (escupidera, tapadera, fregadero, lavadero) y $c$ ) con valor 'acción y efecto de' con un matiz de reiteración (tiradero, gastadero). Se usa de preferencia en el lenguaje familiar y tal vez a esto se deba que sólo documenté un ejemplo: tiradero, donde podríamos suponer (como para -ura) pérdida del morfema de género en el proceso: tirad-o tiradero.

Creo poder afirmar que derivar los sustantivos postverbales aquí tratados de participios constituye una posibilidad válida. Incluso me parece que el hecho de que a los sustantivos que consideré en un principio se hayan venido a unir los en -ado, -a; -ido, -a confirma la presencia del participio también en los en -ción, -miento, etc.

Creo también que para poder situar correctamente los sufijos aquí tratados desde el punto de vista semántico, haría falta tomar en cuenta también los sustantivos en -ancia, -encia, -anza, así como los infinitivos sustantivados, pues compiten en ciertos contextos con los en -ción -ión, -miento, -mento, -ura, - $\phi$, - (a) to, - (a) je, -ero.

Tal vez quepa deducir del grupo más numeroso, formado por los derivados en -ción, una posibilidad de evolución semántica que motiva un continuo ir y venir entre participios y sustantivos: cuando ha dejado de existir el verbo cuyo participio ha dado origen a un sustantivo de los aquí tratados y éste ha pasado a ser nombre de un objeto o concepto, el sustantivo puede, a su vez, servir de base para la formación de un nuevo verbo:

escrito escritura escriturar

escriturado escrituración

fundado fundamento fundamentar

fundamentado fundamentación. 
Está latente, además, para los sufijos aquí tratados el valor 'cualidad de' en los casos en que su base, el participio, puede fungir como adjetivo:

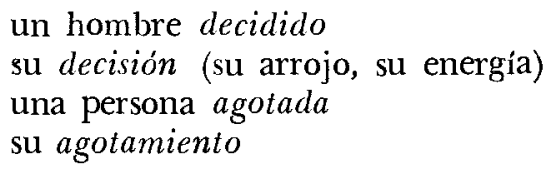

Agotamiento es, al mismo tiempo, efecto de agotarse y cualidad de estar agotado. Esta posibilidad vincula, por lo tanto, a estos sufijos con los que sirven a la formación de sustantivos con base adjetiva, no participial.

El.isabeth Beniers

El Colegio de México. 